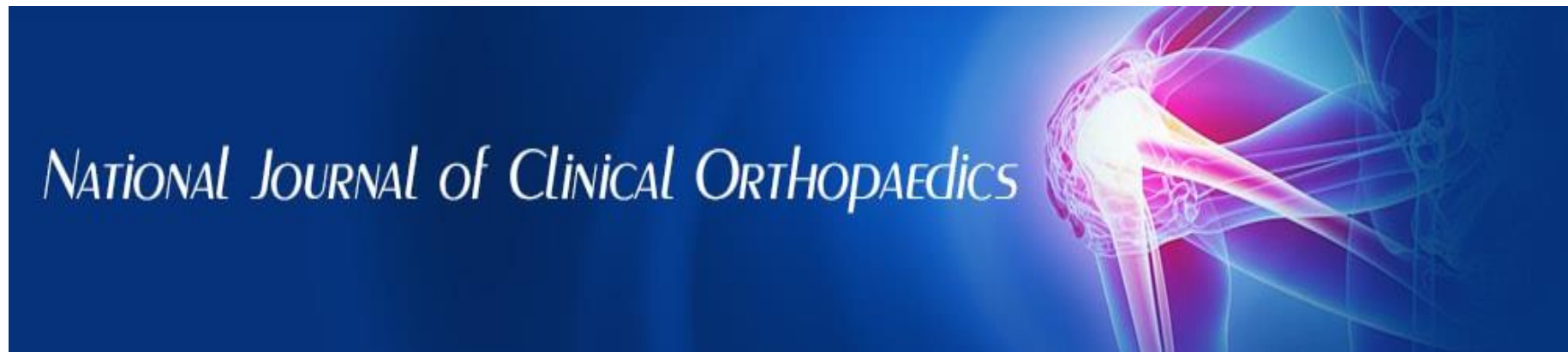

ISSN (P): 2521-3466

ISSN (E): 2521-3474

(C) Clinical Orthopaedics

www.orthoresearchjournal.com

2020; 4(3): 32-37

Received: 16-05-2019

Accepted: 21-06-2019

Dr. Sunil Tarigopula

Assistant Prof, Department Of

Orthopaedics, NRI Academy Of

Sciences, Chinakakani, Guntur

(Dt), Andhra Pradesh, India

Dr. Harsha Nandini Talasila Post Graduate, Department Of Orthopaedics, NRI Academy Of

Scinces, Chinakakani, Guntur (Dt), Andhra Pradesh, India

Dr. Satya Kumar Koduru Prof \& HOD, Department Of Orthopaedics, NRI Academy Of Scinces, Chinakakani, Guntur (Dt), Andhra Pradesh, India

Corresponding Author: Dr. Satya Kumar Koduru Prof \& HOD, Department Of Orthopaedics, NRI Academy Of Scinces, Chinakakani, Guntur (Dt), Andhra Pradesh, India

\section{Study of comminuted unstable intertrochanteric fractures of femur managed with proximal femoral locking plate}

\author{
Dr. Sunil Tarigopula, Dr. Harsha Nandini Talasila and Dr. Satya Kumar \\ Koduru
}

DOI: https://doi.org/10.33545/orthor.2020.v4.i3a.233

\begin{abstract}
Aim \& Objective: The main aim of the present study to investigate the comminuted unstable intertrochanteric fractures of femur managed with proximal femoral locking plate.

Methodology: The present study was conducted at NRI Medical College \& General Hospital, Chinnakakani, Guntur, Andhra Pradesh. During the period of April 2018 to March 2020. All the patients were selected into the study based on inclusion and exclusion criteria. The type of surgery was internal fixation of comminuted unstable intertrochanteric fractures with proximal femoral locking plate. All patients were followed up at 6weeks, 12 weeks, 6, 9, and 12 months.

Results: Out of 30 patients, 20 were males and10 were females. Both anatomical and functional outcomes were assessed in these patients. Out comes were assessed with Harris hip score. The results were good to excellent in $80.01 \%$ of the cases. The majority of injured patients were males $(66.66 \%)$. The average follow up period was 10 months. $60 \%$ of patients do not have any pain during follow-up. 28 of 30 fractures united within a period of 14 months. The average time of union was 14.5 weeks. Four patients had delayed union for whom delayed weight bearing was advised. $95 \%$ of fractures united within 16 weeks. The functional outcome was more that $90 \%$.

Conclusion: Proximal femoral Locking Compression Plate provides the surgeon with flexibility to achieve angular stability or axial compression with the plate to bone apposition.
\end{abstract}

Keywords: Proximal Femoral Locking, intertrochanteric fractures, Axial compression, Angular stability

\section{Introduction}

Intertrochanteric fractures account for nearly 50percent of all fractures of the proximal femur [1]. There are approximately 1,50,000 intertrochanteric fractures annually in the United States, with an annual incidence of 63 and 34 per 100,000 population per year for elderly males and females, respectively.

The ratio of incidence of these fractures in men to women ranges from 1:8 to 1:2, likely because of postmenopausal metabolic changes in bone. These fractures are caused due to high energy trauma in young patients, while in elderly patients, they are due to low energy trauma like slip and fall in osteoporotic bone. Surgical treatment is the preferred modality in the sefracture $\mathrm{s}$ to reduce the complications of prolonged immobilization. Prolonged immobilization leads to deep vein thrombosis, pulmonary embolism, respiratory tract infections, and bedsores. The implants used for fixation of these fractures fall into two main categories, i.e., extramedullary and intramedullary. Extramedullary implants such as dynamic hip screws, dynamic condylar screws, and $95^{\circ}$ condylar blade-plates provide stable fixation in the cancellous bone of the head and neck with adequate rotational stability.

Their demerits are extensive devascularization, longer operating time, higher infection rate, delayed weight-bearing, shortening, medialization of the distal fragment; implant cut-outs, uncontrolled lateralization of the proximal fragment, and varus collapse. Intramedullary fixation, when compared with extramedullary fixation, is associated with short operative time and minimal blood loss and has better biomechanical properties. 
But they have few technical difficulties and complications in comminuted intertrochanteric fractures. Recently, in the treatment of comminuted proximal femur fractures, the proximal femur locking plate is being used. The advantage of Proximal femoral locking plates is that they allow angular stable fixation points into the proximal femur while leaving a smaller 'footprint,' thereby preserving more bone stock than large proximal lag screws.

To evaluate the role of proximal femoral locking plate in comminuted unstable intertrochanteric fractures of the femur and to note functional outcome and complications through follow up of the patients operated upon in NRI General Hospital, Chinakakani, Guntur during the period between April 2018 to March 2020.

\section{Materials and methods}

Thirty Patients with intertrochanteric fractures admitted in NRI General Hospital, Chinakakani, Guntur, during the period between April 2018 TO March 2020, who met the following inclusion criteria were taken up for the study after obtaining consent.

\section{Inclusion criteria}

1. Patients of comminuted unstable intertrochanteric fractures

2. Age group between $20-75$ years

3. Patients with medical fitness for surgery

4. The patient who give consent for the study.

\section{Exclusion criteria}

1. Patient medically unfit for surgery.

2. Patient not willing for surgery

Clearance from the institutional ethical committee was obtained before initiating the study.

On admission, emergency care was given, with particular attention to A (airway), B (breathing), C (circulation). A thorough systematic examination is done to rule out other injuries. They were examined for signs of fractures, deformity, and any compromise of distal neurovascular status. A thorough general examination and local examination was performed.

Radiological examination of the affected part and routine investigations were carried out. Patients were taken up for surgery as early as possible in all the cases. Older people with medical problems after thorough workup were taken up for surgery, once the patient is fit for surgery. Preoperatively all patients were immobilized with skin traction with an attached weight of $3 \mathrm{kgs}$ and elevation of the limb was advised. Associated injuries were dealt with simultaneously or at a later date, depending upon convenience.

Patients with open wounds and associated injuries waited until the conditions permit for surgery. On eurovascular injuries were noted in this series. In patients with severe blood loss and hypovolemic shock, it was corrected with intravenous fluids and blood.

For simple fractures, the antibiotic regimen was started 12 hours before surgery parenterally and continued till the fifth postoperative day; from then till the 10th postoperative day, oral preparation was given. All patients were taken up for surgery when the general condition was stable under spinal anesthesia or general anesthesia.

\section{Implants and instruments}

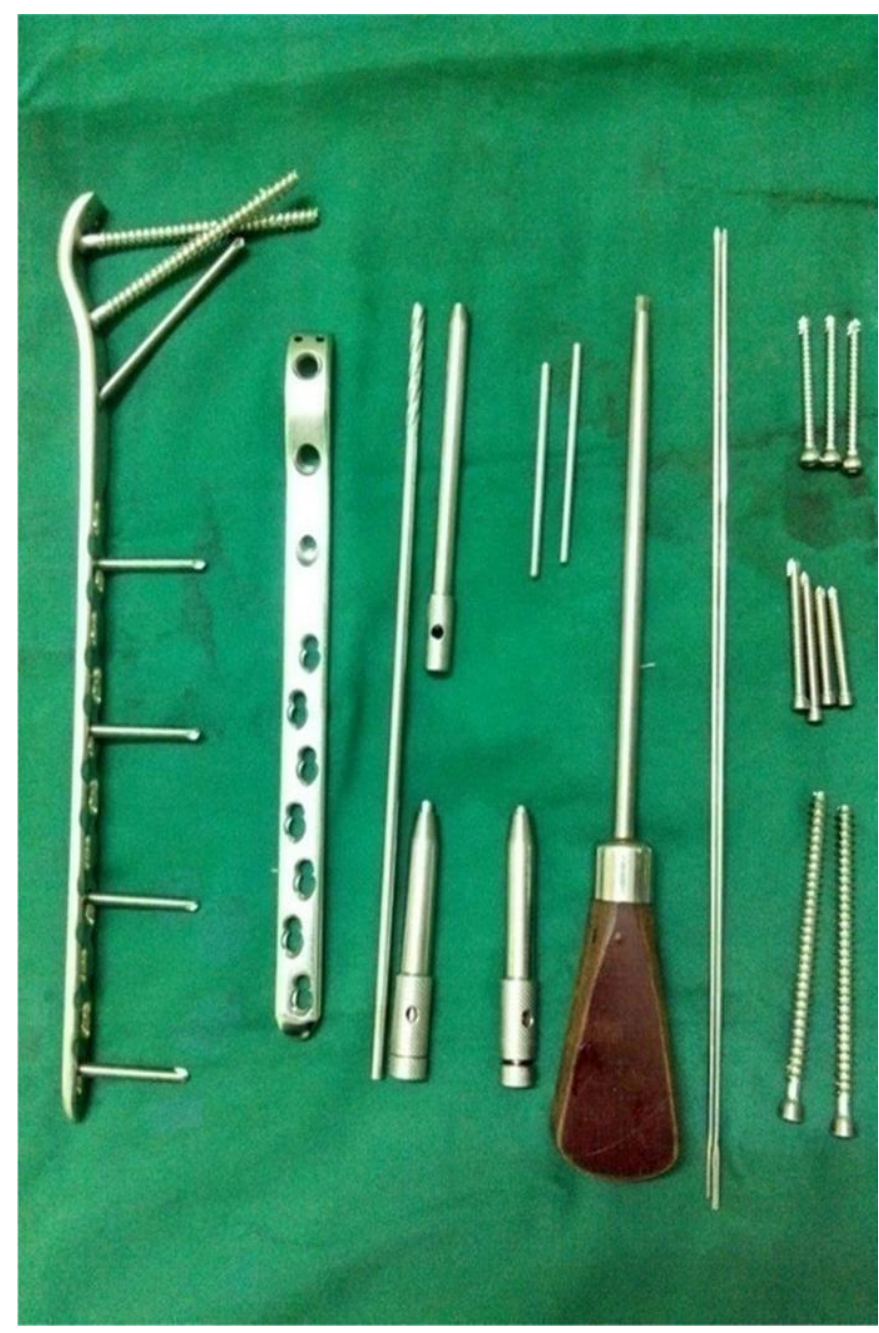

Fig 1: The implants used were proximal femoral locking plate and screws

\section{Patient positioning and fracture reduction}

After induction of anesthesia, the patient was placed in the supine position on the fracture table with the adduction of the affected limb by 10-15degrees, and closed reduction of the fracture was made with traction and rotation. In some instances, the limb was flexed 10-15 degrees to bring the distal fragment in alignment with the proximal fragment. The unaffected limb was abducted as far as possible to accommodate the image intensifier (C -Arm). Alternatively, the limb can be abducted and flexed as the fracture table permits. The image intensifier was positioned so that simultaneous anterior-posterior and lateral views could be taken.

The patients were given as crub, and then parts were painted and draped as for the standard hip fracture fixation. The prophylactic antibiotic was given to all patients at the time of induction.

\section{Approach}

The tip of the greater trochanter was located by palpation or occasionally by using image intensifiers in obese patients. A 6-8 cms vertical incision was taken from the tip of trochanter in the distal direction along the shaft of the femur. Skin and subcutaneous tissue incised. Tensar Fascia lata was opened in line with the incision, and the gluteusmedius and vastuslateralis muscles were split in line with the fibers and tip of the trochanter and proximal femur exposed. Insert guide wires and establish screw trajectories. 
Before placing the plate on the bone, first, thread the wire guides into the plate holes for each of the 3 proximal locking screws. Use the wire guide in the 2 pro ximal screwholes, and a wire guide

In the $3^{\text {rd }}$ locking screw hole. The wire guides can also be used as a manipulation aid for positioning the plate on the proximal femur.

Using fluoroscopic image control (AP and lateral), insert a guide wire through the wire guide in each of the three proximal locking holes. For proper screw measurement, guide wires should reach but not penetrate sub-chondral bone.

Note: It is more important to correctly place guide wires in the proximal femur (considering the desired screw positions) than it is to precisely match the contour of the plate to the anatomy of the femur. The ability to lock the screws to the plate obviates the need for precise plate contouring and compressing the plate to the bone.

Under C-arm guidance, three guide pins passed into the femoral neck at 95 degrees, 120 degrees and 135 degrees. After drilling, proximal fragment fixed with three $6.5 \mathrm{~mm}$ fully threaded cancellous screws and remaining distal holes fixed with $5 \mathrm{~mm}$ cortical screws. Fracture reduction and position of screws confirmed under C-arm.

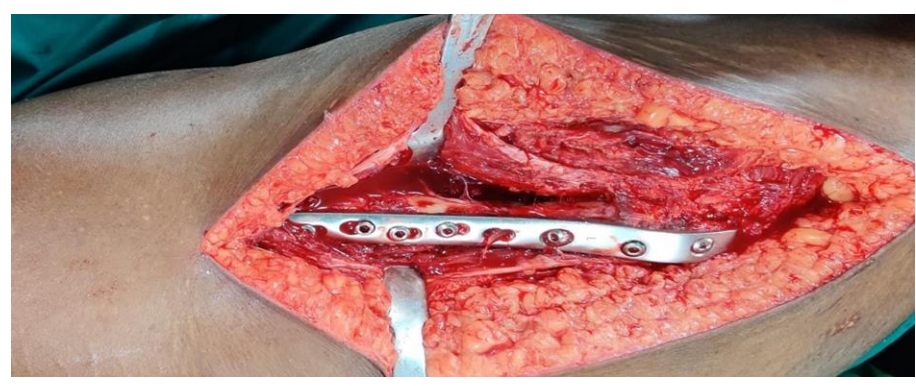

Fig 2. Intraop image

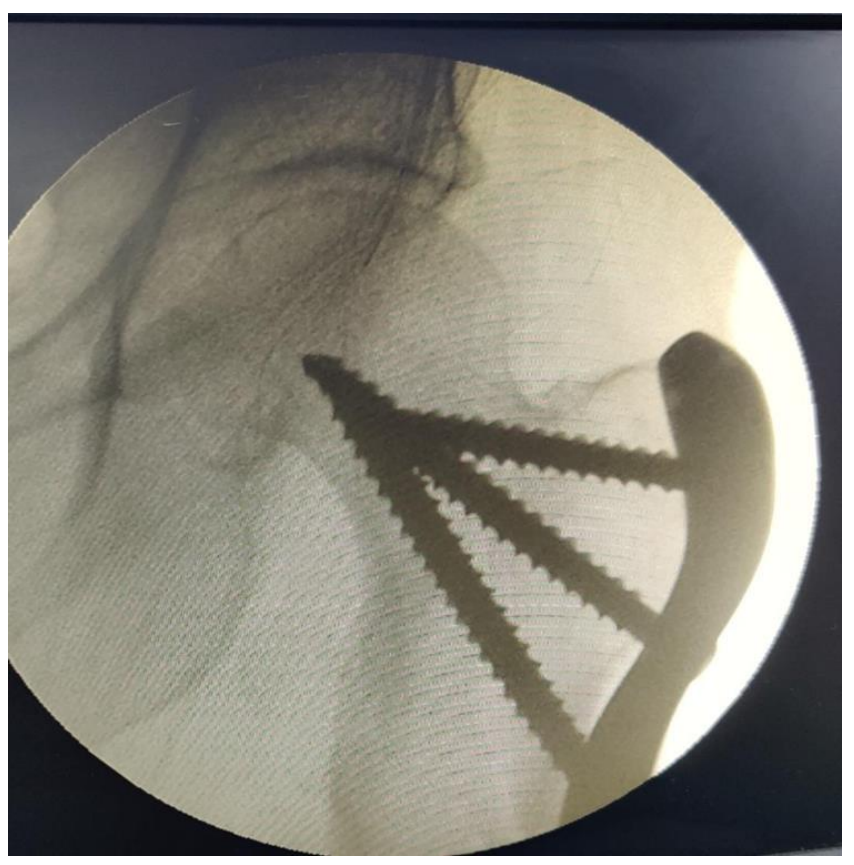

Fig 3: C-ARM Image

\section{Closure}

After the completion of the fixation, a thorough wash of the wound was given with normal saline. A suction drain was inserted at the entry point and wound closed in layers. Sterile dressing was applied, and the compression bandage used

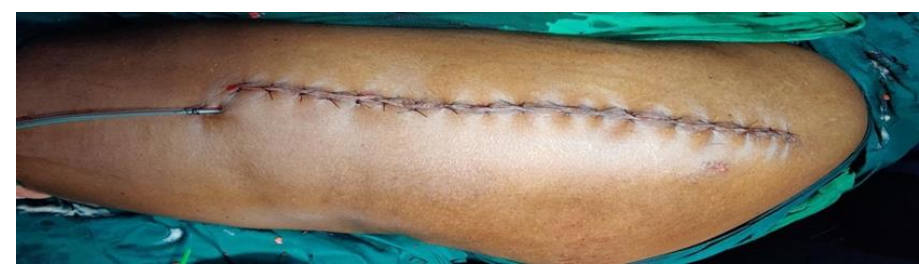

Fig 4: Closer Image

\section{Postoperative care}

The patient's vitals were monitored. Foot end elevation was given overnight. The drain was monitored. Antibiotics were given as per the hospital protocol. Analgesics were given as per patient compliance. Blood transfusion was given depending upon the general preoperative condition and intraoperative blood loss.

Post operative $\mathrm{X}$ ray was taken.

Patients were encouraged to sit in the bed the next day and were taught Quadriceps strengthening exercises and knee mobilization.

Sutures were removed on day 12 or 13. Patients were discharged from the hospital at two weeks with non weight bearing walking on the operated limb with the help of walking aids.

\section{Follow up}

All patients were followed up at 6weeks, 12 weeks, 6, 9, and 12 months. At every visit, the patient was assessed clinically regarding hip and knee function, walking ability, shortening, and fixation defects. X-ray of the involved hip with femur was done to evaluate fracture union and implant-bone interaction. The functional outcome of the patient is assessed by a harris hip score whose components are as follows.

\section{Duration of hospitalization}

The average duration of hospital stay following surgery was ten days ranging from 7-14 days.

\section{Mortality}

There was no mortality in this series of case study

\section{Follow Up}

The average duration of follow up was 12 months, ranging from 6-16 months, of which all the patients were available for follow up.

\section{Radiological Union}

The radiological union was said to be achieved on the evidence of obliteration of fracture lines and trabecular continuity between the two fragments on anteroposterior and lateral $\mathrm{x}$ rays in three cortices.

The union is considered delayed when healing has not advanced at the average rate for the location and type of fracture.

Presence of callus for evaluation of fracture union and complications (implant cut-out and varus collapse) assessed on radiographs. Hip function assessed using the Hospital for Special Surgery: Hip Rating System ${ }^{[37] .}$

\section{Results}

In the past two years from April 2018 to March 2020, 30 cases of comminuted unstable intertrochanteric fractures of femur treated in the NRI Academy of medical sciences.

The analysis is as follows. 


\section{Age distribution}

Table 1: Age distribution

\begin{tabular}{|c|c|c|}
\hline Age & No. of patients & \% \\
\hline $20-30$ & 6 & 20 \\
\hline $31-40$ & 4 & 13.33 \\
\hline $41-50$ & 8 & 26.66 \\
\hline $51-75$ & 12 & 40 \\
\hline
\end{tabular}

The age of the patients ranged from 20 years to 75 years. The mean age of the patients was 47.5 years.

\section{Sex distribution}

Table 2: Sex distribution

\begin{tabular}{|c|c|c|}
\hline Sex & No. of Patients & \% \\
\hline Males & 20 & 66.66 \\
\hline Females & 10 & 33.33 \\
\hline
\end{tabular}

Among the 30 patients, 20 were males, and 10 patients were females

Table 3: Mode of Injury

\begin{tabular}{|c|c|c|}
\hline Mode of Injury & No. of Patients & \% \\
\hline Fall at ground level & 20 & 66.66 \\
\hline Road traffic acciden & 10 & 33.33 \\
\hline
\end{tabular}

The mode of injury was fall at ground level in 20 patients and road traffic accidents in 10 patients.

\section{Side involved}

Table 4: Side involved

\begin{tabular}{|c|c|c|}
\hline Side & No. of patients & \% \\
\hline Right & 20 & 66.66 \\
\hline Left & 10 & 33.33 \\
\hline
\end{tabular}

12 patients had a right-sided injury and 8 patients had left-sided injury

\section{Associated injuries}

4 out of 30 patients had associated injuries of other bones. 1 patient had clavicle fracture which was treated conservatively with clavicle brace. 2 patients had distal radius fractures which were treated with percutaneous $\mathrm{K}$ wire fixation. 1 patient had a subtrochanteric fracture of the opposite femur which was treated with Long Proximal Femur Nailing.

Table 5: Associated Injuries

\begin{tabular}{|c|c|c|}
\hline Injury & No of Patients & \% \\
\hline Clavicle Fracture & 1 & $3.33 \%$ \\
\hline Distal radius fracture & 2 & $6.66 \%$ \\
\hline Subtrochanteric Fracture of the opposite femur & 1 & $3.33 \%$ \\
\hline
\end{tabular}

Table 6: Type of fractures

\begin{tabular}{|c|c|c|}
\hline Boyd and Griffin type of fracture & No. of patients & $\%$ \\
\hline Type 3 3 & 12 \\
\hline Type 4 & 18 & 60 \\
\hline
\end{tabular}

12 patients had Type 3 Boyd and Griffin, and 8 patients had Type 4 Boyd and Griffin fracture

\section{Anatomical results}

Anatomical results were assessed by the presence or absence of shortening, range of movements, and deformities. $80 \%$ of the cases had good results, and $20 \%$ had poor results.

Table 7: Anatomic results

\begin{tabular}{|c|c|c|}
\hline Results & No. of cases & \% \\
\hline Good & 24 & 80 \\
\hline Poor & 6 & 20 \\
\hline
\end{tabular}

\section{Functional results}

Functional results were assessed in the 30 cases available for follow up using Harris hip score.

Excellent results were noted in 6 cases, good in 20 cases, fair in 2 cases, and poor in 4 cases.

- Harris hip score above $90 \%$ is Excellent

- Harris hip score above $80 \%$ is Good

- Harris hip score above $70 \%$ is Fair

- Harris hip score above $60 \%$ is poor

Table 8: Functional results

\begin{tabular}{|c|c|c|}
\hline Results & No. of cases & \% \\
\hline Excellent & 6 & 20 \\
\hline Good & 18 & 60.01 \\
\hline Fair & 2 & 6.66 \\
\hline Poor & 4 & 13.33 \\
\hline
\end{tabular}

\section{Early complications}

Early complications were encountered in 3(10\%) patients.

- 4 patients had wound gaping andinfection.

- Out of them, 2 patients had diabetes mellitus required secondary suturing after glycemic control.

- The other 2 patients were treated immediately with a wound wash and with appropriate antibiotics.

Table 9: Early Complications

\begin{tabular}{|c|c|c|}
\hline Complication & No of patients & \% \\
\hline Wound gaping & 4 & 13.33 \\
\hline Infection & 4 & 13.33 \\
\hline Nil & 26 & 86.66 \\
\hline
\end{tabular}

Table 10: Late complications

\begin{tabular}{|c|c|c|}
\hline Complications & No of Patients & $\%$ \\
\hline Proximal screw back out & 2 & 6.66 \\
\hline Varus collapse & 4 & 13.33 \\
\hline Delayed union & 4 & 13.33 \\
\hline Nil & 2 & 66.66 \\
\hline
\end{tabular}

The late complications were seen in 10 patients. 2 patients had proximal screw backout, 4 patients had varus collapse and 4 patients had delayed union of the fracture.

\section{Discussion}

Proximal femoral fractures are a significant challenge by the orthopaedic community, in achieving fracture union and restoration of function with minimum complications.

Intertrochanteric fractures tend to unite due to their vascularity, metaphyseal and extra synovial location. However, they result in malunion, resulting in shortening, varus, medialisation of the shaft, and external rotation deformity. Thus, it is necessary to achieve near-anatomic reduction and maintain it till union, but this is not possible when a DHS is used, as intra- and postoperative collapse may occur and lead to shortening or 
medialisation of the shaft, especially in unstable intertrochanteric fractures, including fractures with a large posteromedial void, reverse oblique fractures with subtrochanteric extension, and fractures with loss of lateral buttress (greater trochanter). Osteoporotic hips are at high risk of instability and comminution.

Evans classified intertrochanteric fractures based on the stability of the fracture pattern: stable and unstable types.

- Unstable fractures are those, which are displaced and cannot be reduced, postero- medial cortical comminution, and reverse oblique fractures.

- Stableintertrochantericfractureshavecorticalcontinuityafterre duction, without a gap medially, posteriorly, andlaterally. This contact prevents displacement of fracture in to varus or retroversion when forces are applied to the proximalfemur.

However, in unstable intertrochanteric fractures, the reverse oblique pattern and medial displacement of the shaft tends to occur due to adductor muscle pull.

The aim of the management of intertrochanteric fractures is to achieve early mobilization, rehabilitation, and quick return of individuals to daily activities. The early operative intervention of intertrochanteric fractures reduces both mortality and morbidity (Hasenboehler et al.) ${ }^{[2]}$, giving the best chance of early indepen dency and minimizing the risks of prolonged bed rest.

The implants used for fixation of these fractures fall into two main categories, i.e., extramedullary and intramedullary. Extramedullary implants such as dynamic hip screws, dynamic condylar screws, and $95^{\circ}$ condylar blade-plates provide stable fixation in the cancellous bone of the head and neck with adequate rotational stability.

Their demerits are extensive devascularization, longer operating time, higher infection rate, delayed weight-bearing, shortening, medialization of the distal fragment, implant cut-outs, uncontrolled lateralization of the proximal fragment, and varus collapse. Intramedullary fixation, when compared with extramedullary fixation, is associated with short operative time and minimal blood loss and has better biomechanical properties. But they have few technical difficulties and complications in comminuted intertrochanteric fractures.

Recently, in the treatment of comminuted proximal femur fractures, the proximal femur locking plate is being used. The management plan for unstable intertrochanteric fractures differs from that for stable fractures. Using an implant with a trochanteric flange decreases the chance of lateralisation of the proximal fragment, thereby improving the abductor lever arm. A locking plate helps to maintain reduction until union with less risk of limb shortening or varuscollapse because it is a noncollapsing.

Early stabilization of unstable intertrochanteric fractures with mechanically more stable implants enables earlier weightbearing, recovery of ambulatory function. This prevents complications of prolonged immobilization like deep vein thrombosis, pulmonary embolism, respiratory tract infections, and decubitus ulcers. Unstable intertrochanteric fractures include are those fractures with a large posteromedial void, reverse oblique fractures with subtrochanteric extension, and fractures with loss of lateral buttress (greater trochanter comminution) [3].

It is crucial to achieve near anatomic reduction and maintain it till fracture union, which is not feasible with implant like a dynamic hip screw, as intraoperative and post-operative collapse may occur and lead to shortening or medialisation of the diaphysis ${ }^{[4]}$.There may be screw back out of the DHS side plate, owing to increased stresses at the screw plate junction. This complication can be avoided by using an implant with a locking neck and shaft screws, which are non-collapsing. The greater trochanter is the only structure that resists. The lateralization of the proximal fragment. Using a dynamic hip screw system in a fracture without a lateral but tress inevitably leads to medialisation of the diaphysis, non-union, and screw cut-out. In such fractures non-collapsing proximal femur locking plate is useful.

Dynamic sliding hip screw system and intramedullary implant like proximal femoral nailanti- rotation system enable controlled impaction of the intertrochanteric fracture fragments. In contrast, the PF-LCP system locks the fracture in position without a controlled collapse.

The use of a single large Dynamic hip lag screw necessitates the reaming of lots of healthy cancel lous bone, which is detrimental in osteoporotic hips. According to the literature review, the volume of bone reamed was $8586 \mathrm{~mm} 3$ for one dynamic condylar screw or DHS lag screw $(12.5 \mathrm{~mm}$ in diameter, $70 \mathrm{~mm}$ in length) and $6965 \mathrm{~mm} 3$ for three locking screws (each $6.5 \mathrm{~mm}$ in diameter, $70 \mathrm{~mm}$ in length) placed through the proximal femoral locking plate. More bone stock is preserved in fixation using a locking plate.

When a single Dynamic hip lag screw is used in the fixation, there is either excessive co- axial collapse in unstable intertrochanteric fractures without a lateral cortex or excessive bending stress at the lag screw-plate junction.

In contrast, multi-directional locking screws decrease the stress concentrated at the screw- plate junction with a $95^{\circ}$ screw preventing the collapse. Even with the implant like a trochanteric lateral but tressplate, there is excess stress on the plate screws that prevent medialisation. Hence, locking screws at the screw plate construct is necessary.

The chance of limb shortening is common when using a Dynamic hip screw system, as co- axial collapse along the barrel occurs due to the proximal metaphyseal posteromedial void. The use of a locking plate prevents the collapse. In unstable intertrochanteric fractures with a large posteromedial void treated with a DHS, a varus collapse could occur, due to the implant break off at the neck or the DHS lag screw toggling within the head.

The proximal femoral locking plate is designed to have a lateral trochanteric buttress to prevent lateralization of the proximal fragment. The proximal femoral locking compression plate is fixed with many multi-directional smaller diameter screws to hold the head at $95^{\circ}$ and $135^{\circ}$ directions. This allows us to preserve more cancellous bone. The PFLCP was reported to be the strongest construct for vertically orientated femoralneck fractures. The PF-LCP with the 'kickstand' screw was reported to have similar biomechanical properties as the $95^{\circ}$-angled blade plate.

The amount of medialisation of diaphysis determines the length of the abductor lever arm. With medialisation of the shaft, the length of the abductor lever arm decreases and leads to an abductor lurch and affects functional outcome. The use of PFLP prevents medialisation, thereby reducing the occurrence of abductor lurch.

The PFLP, along with kickstand screw is biomechanically equivalent to the angled blade plate. The PFLCP provided angular stability with a higher degree of adjustment compared with the angle dbladeplate and of fered the same function while avoiding excessive bone removal compared with the dynamic condylar screw. PF-LCP fixation is appropriate for complex proximal femoral fracture fixation. 
Table 11: Mean age comparision with other studies

\begin{tabular}{|c|c|}
\hline Study & Mean age \\
\hline Our study & 47.5 \\
\hline Wang et al. ${ }^{[5]}$ & $73 \mathrm{yrs}$ \\
\hline Balter et $^{[6]}{ }^{[6]}$ & $67 \mathrm{yrs}$ \\
\hline
\end{tabular}

In our study, the common mode of injury being trivial fall accounting for $66.66 \%$ of the cases. Wang et al. ${ }^{[5]}$ and Balter et al. [6] states low energy trauma due to fall being the most common mode in intertrochanteric fractures in elderly and highvelocity injuries in young adults.

Table 12: Mode of Injuries compared with previous ones

\begin{tabular}{|c|c|}
\hline Study & Mode of Injury \\
\hline Our study & Trivial injury \\
\hline Wang, et al. $^{[5]}$ & $\begin{array}{c}\text { Low energy trauma in elderly high } \\
\text { velocity injury in adults }\end{array}$ \\
\hline Balter et al. ${ }^{[6]}$ & $\begin{array}{c}\text { Low energy trauma in elderly high } \\
\text { velocity injury in adults }\end{array}$ \\
\hline
\end{tabular}

In our study, there were difficulties in placing all the three proximal screws into the head through the neck in 2 cases and also placement of the plate along the shaft in 2 cases. This is attributed to the pre-contouring of the plate and different neck shaft angle and ante-version in individual patients and also due to low to moderate built of the patients in our region.

The average length of incision in our study is $7 \mathrm{cms}$ with bigger incision needed in comminuted fractures with subtrochanteric extension. The length of the plate is 5 holes in the case of intertrochanteric fractures and eight holes in fractures extending to the subtrochanteric region.

In our study, 50 percent of the cases showed bony union at three months follows up, and 80 percent of cases showing bony union at six months follow up. Four cases showed delayed union, and two patients had proximal screw back out, and four patients had varus collapse.

In our study, $80.01 \%$ of fractures achieved union, $19.99 \%$ of fractures had complications. Zha GC, Chen ZL et al. ${ }^{[7]}$ reports a union rate of $95 \%$ and $98 \%$ at three months, and six months follow up, respectively.

Table 13: Comparision of Union rate with previous findings

\begin{tabular}{|c|c|}
\hline Study & Union Rate \\
\hline Our study & $80.1 \%$ \\
\hline Zha GC, Chen ZL et al. & $95 \%$ \\
\hline
\end{tabular}

Compare with excellent results from previous studies $(95-100 \%$ union rate) with less complication (1.8-12.5\%). The complications in this study are probably due to poor bone quality, early weight-bearing of the patient as well as lack of application of kickstand screw. The kickstand screw played an essential role in preventing the varus collapse of the construct.

To achieve good results from the Proximal locking compression plate, the surgeon should focus on adequate plate length, nearanatomical reduction with or without a circumferential wire in subtrochanteric fractures, a good medial buttress of the fracture site, use of kickstand screw, biologic friendly or submuscular insertion of plate whenever possible.

\section{Conclusion}

A proximal femur fracture is common in the elderly due to osteoporosis and in young dueto high-velocity trauma. As the fracture is more common in the elderly, early reduction and internal fixation increases patient comfort, facilitates nursing care, helps in early mobilization of the patient, and decreases the duration of hospitalization. As the incidence of comminution is high, these fractures may require a stable reduction and internal fixation. Proximal femoral LCP is a suitable method for proximal femur fractures in elderly patients, especially for severely comminuted fracture and osteoporosis. Proximal femoral Locking Compression Plate provides the surgeon with flexibility to achieve angular stability or axial compression with the plate to bone apposition.

A few of the disadvantages associated with PFLC Parerelatively difficult to perative technique and mechanical implant failure with varus collapse. In light of these observations, we conclude that the Proximal femoral LCP, despite a few unfavourable results and complications, it is a satisfactory method of surgical management in proximal femur fractures with severe comminution and osteoporosis. It requires closed monitoring during the preoperative, intra operative, post-operative period to avoid complications that can be managed.

\section{Acknowledgment}

The author is thankful to Department of Orthopaedics for providing all the facilities to carry out this work

\section{Conflict of Interest \\ None}

\section{References}

1. Koval KJ, Zuckermann JD. Handbook of Fractures, Lippincott Williams \& Wilkins, 3rd edition, 2006.

2. Hasenboehler EA, Agudelo JF, Morgan SJ, Smith WR, Hak DJ, Stahel PF. Treatment of complex proximal femoral fractures with the proximal femur locking compression plate. Orthopedics. 2007; 30(8):618-23.

3. Wieser K, Babst R. Fixation failure of the LCP proximal femoral plate 4.5/5.0 in patients with missing posteromedial support in unstable per-, inter-, and subtrochanteric fractures of the proximal femur. ArchOrthop Trauma Surg. 2010; 130(10):281-7.

4. Kinast C, Bolhofner BR, Mast JW, Ganz R. "Sub trochanteric fractures of the femur: results of treatment with the 95-degree condylar blade-plate" Clin Orthop. 1989; 238:122

5. Wang Y, Yang YY, Yu ZH, Li CQ, Wu YS, Zheng XX. Comparative study of intertrochanteric fractures treated with proximal femur locking compress plate in aged. ZhongguoGu Shang. 2011; 24(5):370-3.

6. Blatter G, Janssen M, Dousa P, Bartonícek J, Jehlicka D, Skála-Rosenbaum J. "Treatment of subtrochanteric fractures with AO dynamic condylarscrew”. J Injury. 1993; 24(2):90-2.

7. Guo-Chun Zhaa b, Ze-Lin Chenb, et al. Treatment of pertrochanteric fractures with a proximal femur locking compression plate, Injury, Int. J Care Injured. 2011; 42:1294-99. 